\title{
Hallazgos preliminares sobre la eficacia del programa Crianza Reflexiva para cuidadores de niños entre 4 y 12 años de edad
}

\author{
Alkabes-Esquenazi, Louise \\ Universidad Católica Santa María la Antigua, Centro Mentalizar Panamá \\ Panamá, Panamá \\ Louise.esquenazi@gmail.com, 0000-0001-5003-8961 \\ Díaz-Hernández, Rubén \\ Universidad Católica Santa María la Antigua \\ Panamá, Panamá \\ rdiaz@usma.ac.pa.com, 0000-0002-9486-295X
}

Zapata, Johana

Centro Mentalizar Panamá

Panamá, Panamá

johanazapatah@gmail.com

\section{González Flores, Sergio}

Instituto Nacional de Salud Mental

Panamá, Panamá

sergiof-04@hotmail.com

Redfern, Sheila

Anna Freud Centre

London, UK

Sheila.redfern@annafreud.org

\section{Oviedo, Diana C.}

Universidad Católica Santa María la Antigua, Centro de Neurociencias y Unidad de Investigación Clínica,INDICASAT-AIP, Sistema Nacional de Investigación, (SNI) SENACYT

Panamá, Panamá

d.ovi@hotmail.com, 0000-0003-1987-5205 


\section{Abstract}

The Reflective Parenting Program is an intervention to promote caregivers' connection, curiosity about the inner world and enjoyment in interactions with their children. Caregivers, being aware of the mental states of their children and their own, will cultivate the reflective function and/or mentalization and their emotional security. The objective of this research is to measure the effectiveness of the Reflective Parenting Program in caregivers of children aged 4 to 12, in Panama City. A randomized controlled trial was carried out with three measurements, a pre-test, a post-test and a follow-up at three months. A sample of forty caregivers of children between 4 and 12 years of age were included in the study and randomly assigned to one of two groups: control $(n=20)$ and experimental $(n=20)$. At the three measurement moments, the participants completed self-report questionnaires to measure parental stress (PSI-SF), parental efficacy (BPES), parental reflective function (PRFQ) and children's emotional regulation (SDQ and ERC). Preliminary results obtained through mixed effects models allow us to identify that the reflective parenting program had a significant effect on parents' interest and curiosity about their children's mental states, on parental stress, and on externalizing problems of their children. The effects were maintained at three months of follow-up. These findings provide evidence on the effectiveness of the Reflective Parenting program.

Keywords: mentalization, reflective parenting, externalizing problems, parental stress, parenting intervention programs.

\section{Resumen}

El Programa Crianza Reflexiva es un método para fomentar en los cuidadores, una mejor conexión, una mayor curiosidad en el mundo interno de los niños y un mayor disfrute de las interacciones con sus hijos. Los cuidadores, al estar conscientes de los estados mentales de sus hijos y de los suyos propios, cultivan la función reflexiva y/o mentalización y la seguridad emocional de ellos. El objetivo de esta investigación es medir la efectividad del programa Crianza Reflexiva en cuidadores de niños de 4 a 12 años, en la ciudad de Panamá. Se realizó un ensayo controlado aleatorizado con tres mediciones, un pre-test, una post-test y un follow-up a tres meses. Una muestra de cuarenta cuidadores de niños entre 4 y 12 años de edad, fueron incluidos en el estudio y asignados de manera aleatoria a uno de dos grupos: control $(n=20)$ y experimental $(n=20)$. En los tres momentos de medición los participantes completaron cuestionarios de autoreporte para medir el estrés parental (PSI-SF), la eficacia parental (BPES), la función reflexiva parental (PRFQ) y la regulación emocional de niños (SDQ y ERC). Los resultados preliminares obtenidos mediante modelos de efectos mixtos permiten identificar que el programa de crianza reflexiva tuvo un efecto significativo, 
sobre el interés y curiosidad de los padres acerca de los estados mentales de sus hijos, sobre el estrés parental, y sobre los problemas externalizantes de sus hijos. Los efectos se mantuvieron a los tres meses de seguimiento. Estos hallazgos aportan evidencia sobre la efectividad del programa Crianza Reflexiva.

Palabras claves: mentalización, crianza reflexiva, problemas externalizantes, estrés parental, programas de intervención para padres.

\section{INTRODUCCIÓN}

En el 2018 en Panamá, el 70\% de los casos que fueron atendidos en los Juzgados de Niñez y Adolescencia por casos de protección, ingresaron por situaciones de abandono, descuido o negligencia por parte de los progenitores[1]. Gran parte de esta población ha sido víctima de disciplina violenta. Una posible explicación del uso de disciplina violenta, es que la desconexión emocional de los padres puede causar comportamientos de negligencia emocional o violencia física por medio del castigo u otro tipo de maltrato a sus hijos[2], [3]. Esto implica un alto costo económico y social ya que es la violencia un factor de riesgo para salud mental y en la constitución de relaciones interpersonales de los niños. La evidencia científica disponible, ha señalado que las intervenciones parentales tienen efectos positivos en el estrés parental y la regulación emocional de los niños[4], [5]. El Programa Crianza Reflexiva[6], [7] es un método para fomentar en los cuidadores, una mejor conexión, una mayor curiosidad en el mundo interno de los niños y un mayor disfrute de las interacciones con sus hijos. Los cuidadores, al estar consciente de los estados mentales de sus hijos y de los suyos propios, cultivan la función reflexiva y la seguridad emocional de ellos[8]-[11].

\section{MÉTODO}

\section{A. Tipo de Estudio}

Se utilizó un diseño experimental, desde un enfoque cuantitativo, con el objetivo de explicar los efectos del programa RPP. El diseño incluyó dos cohortes (tratamiento y control de lista de espera).

\section{B. Participantes}

Se inscribieron 40 cuidadores que reunían las características de los criterios de inclusión. Los participantes debían tener hijos entre 4 y 12 años de edad. La edad promedio de los cuidadores fue de 37.5 años. El 60\% $(n=40)$ de los participantes estaba casado. El $57 \%(n=40)$ tenía una licenciatura o completado estudios universitarios. El $46 \%(n=40)$ de los participantes tenía trabajo de tiempo completo. El 52\% $(n=40)$ de los participantes tenían hijos varones y el promedio de edad de los hijos era de 7 años de edad.

\section{Tratamiento}


El RPP es una intervención psicoeducativa manualizada para mejorar la crianza, a través del foco en la mentalización de los padres. Consta de 8 sesiones cada una con un objetivo específico y con una duración de dos horas, en las que se desarrollan actividades que incluyen la explicación de conceptos, la discusión de ejemplos prácticos, el uso de juego de roles y la distribución de herramientas para trabajar en casa. La intervención está originalmente planificada para desarrollarse de manera presencial pero tuvo que implementarse de manera virtual para ajustarse a las condiciones de la pandemia por COVID-19. Estas adaptaciones incluyeron hacer las reuniones por la plataforma de zoom y utilizar los "breakout rooms" para los juego de roles.

\section{Procedimiento}

Se mandó un afiche en las redes y luego los participantes contactaron a la investigadora directamente a su número de celular. Se verificó si formaban parte de los criterios de inclusión y se les explicó el estudio. Al aceptar la participación en el ensayo, completaron el proceso del consentimiento informado y llenaron los 6 cuestionarios de autoreporte. Al llenar la primeda medida, el pre-test (T1) se le entregó un sobre cerrado que se realizó con la técnica de SNOSE (Sequentially numbered, opaque sealed envelope). Este es un método accesible de asignación oculta, que no necesita del uso de la tecnología es efectiva y de bajo costo. Luego cada participante tomó un sobre y se asignó a su respectivo grupo, tratamiento o lista de espera. Se conformaron dos grupos de tratamiento y dos grupos de lista de espera (control) con 10 participantes cada uno. La duración de este estudio fue de 20 semanas. Se inició con las 8 semanas del programa psicoeducativo y al concluirlas se hizo el post-test (T2). En la semana 12 se hizo un follow up de las herramientas y luego a los tres meses de hacer la (T2) se hizo un post-post test (T3).

\section{E. Mediciones}

Se utilizaron cuestionarios de autoreporte para recolectar los datos de los participantes. En total se aplicaron 6 instrumentos: un cuestionario ad-hoc para recoger datos sociodemográficos; el (PSI-SF) Parenting Stress Index Short Form [10] para el Estrés Parental; el (BPES) Brief Parental Efficacy Scale[11] para la Eficacia Parental, el (PRFQ) Parental Reflexive Function Questionnaire[12] para la Función Reflexiva Parental; el (SDQ) Strenghts and Difficulties Questionnaire[13] y el (ERC) Emotional Regulation Checklist[14] para la Regulación Emocional de los niños.

\section{RESULTADOS}

Para determinar la homogeneidad entre los grupos, se compararon las cohortes en función de los datos demográficos recolectados y en las puntuaciones de los cuestionarios de autorreporte mediante una regresión logística bayesiana. La variable dependiente fue el 
grupo asignado (tratamiento o control) y se incluyeron como predictores datos demográficos y puntajes de línea base (T1). Para regularizar los coeficientes de regresión, se definió la probabilidad a priori de herradura[15].

Ninguno de los predictores considerados tiene puede ser considerado como significativo, pues la distribución posterior de cada parámetro se solapa de manera importante (\% en ROPE > 80.0) con la región de interés práctico (ROPE $[-0.18,0.18]$ ), lo que indica que el procedimiento de asignación aleatoria fue efectivo para crear grupos homogéneos.

Considerando que el tamaño de la muestra pudo haber influido en no encontrar coeficientes significativos en la regresión logística, se calculó el puntaje de poder predictivo (PPS)[16]. Se encontró que la edad de los participantes tiene un poder predictivo de $30 \%$ para la asignación al grupo, por lo que se decidió controlar por esta variable en los análisis subsiguientes.

Para evaluar el efecto del tratamiento sobre las variables de interés se estimaron modelos de regresión jerárquica. Para cada resultado (estrés parental, mentalización, regulación emocional), en el primer nivel se incluyeron como dependientes las mediciones en los tres momentos (pre-test, post-test, seguimiento a 3 meses). Como predictores de segundo nivel se incluyeron el tiempo, el tratamiento y la interacción entre ellos. El intercepto se estimó como efecto aleatorio. La significancia estadística del coeficiente de la interacción tiempo $x$ tratamiento se consideró como criterio para determinar que el tratamiento tuvo efectos. En los casos en los que se encontró un efecto significativo, se exploró la interacción mediante análisis de efectos simples para determinar cuáles fueron las diferencias entre los grupos: dirección o magnitud.

Tabla 1 Resumen del efeto del tratamiento sobre la evolución $(\mathrm{T} 1, \mathrm{T2}, \mathrm{T3})$ de los efectos evaluados

\begin{tabular}{|l|c|c|c|}
\hline & & DIRECCION DEL EFECTO \\
\hline & GRUPO CONTROL & $\begin{array}{c}\text { GRUPO } \\
\text { EXPERIMENTALERENCIA } \\
\text { SIGNIFICATIVA }\end{array}$ \\
\hline Efectos en los padres & & \\
\hline Estrés parental & Aumenta & Disminuye & SI \\
\hline Pre-mentalización & Sin Cambios & Sin Cambios & NO \\
\hline Certeza de los estados mentales & Sin Cambios & Sin Cambios & NO \\
\hline Interés y curiosidad en los estados mentales & Disminuye & Sin Cambios \\
\hline Eficacia parental & Sin Cambios & Sin Cambios & NI \\
\hline Efectos en los hijos & & & Aumenta \\
\hline Regulación emocional & Aumenta & Si \\
\hline Liabilidad / Negatividad & Sin Cambios & Sin Cambios \\
\hline Problemas conductuales y emocionales & Sin Cambios & Sin Cambios \\
\hline Problemas externalizantes & Sin Cambios & Disminuye \\
\hline Problemas Internalizantes & Sin Cambios & Sin Cambios \\
\hline
\end{tabular}

En la tabla 1 se resume los resultados de este análisis. En la columna de diferencia significativa se indica si el efecto de la interacción fue significativo (Sí). Aparecen en verde aquellos resultados que se observaron en la dirección esperada; y en rojo, aquellos en la dirección contraria a la esperada. 
Fig.1 Interacciones significativas de tratamiento y tiempo sobre efectos evaluados en los padres

A.

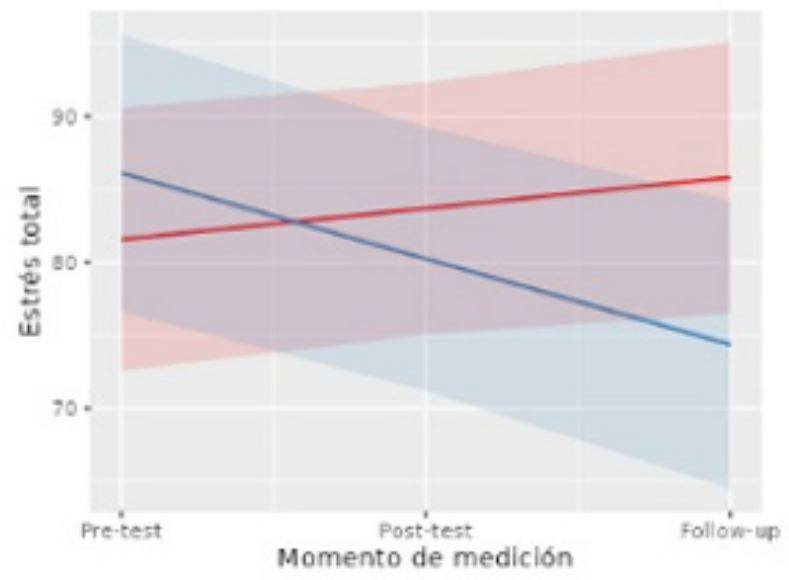

B.

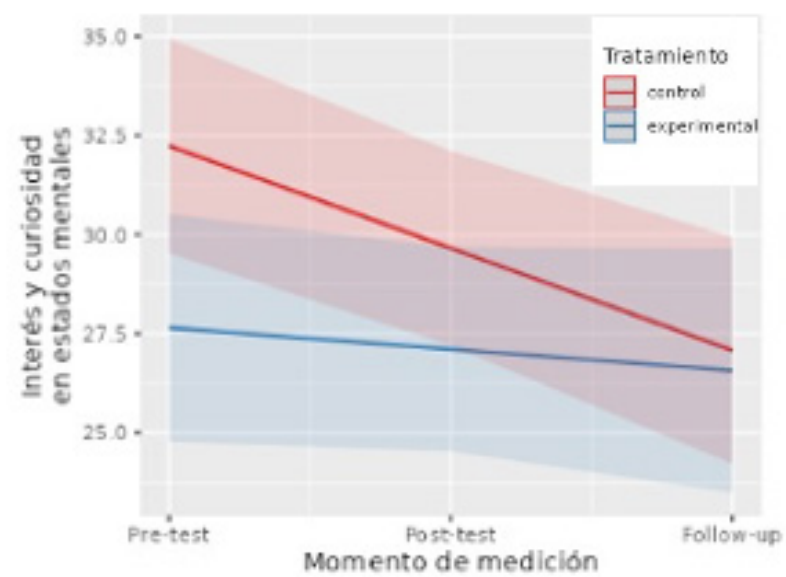

Se observa una disminución significativa del distrés parental en el grupo experimental, así como el mantenimiento de la curiosidad e interés sobre los estados mentales de sus hijos a lo largo de los tres momentos de medición.

La intervención fue efectiva para reducir el estrés parental (Figura 1A), mantener el interés y curiosidad en los estados mentales (Figura 1B) y reducir los problemas externalizantes de los niños (Figura 2A). Contrario a lo esperado, en el grupo control aumentó la regulación emocional de los niños en mayor magnitud que en el grupo de tratamiento (Figura 2B)

Fig. 2 Interacciones significativas de tratamiento y tiempo sobre efectos evaluados en los niños.

A.

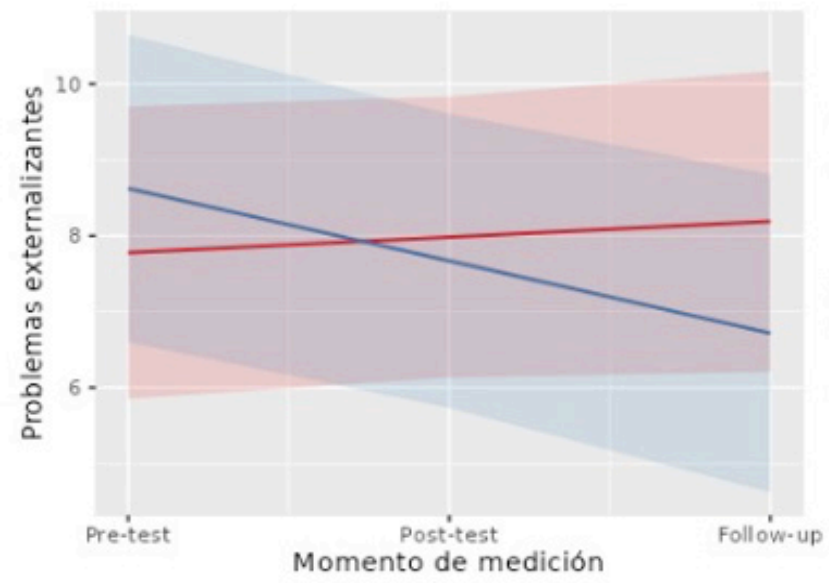

B.

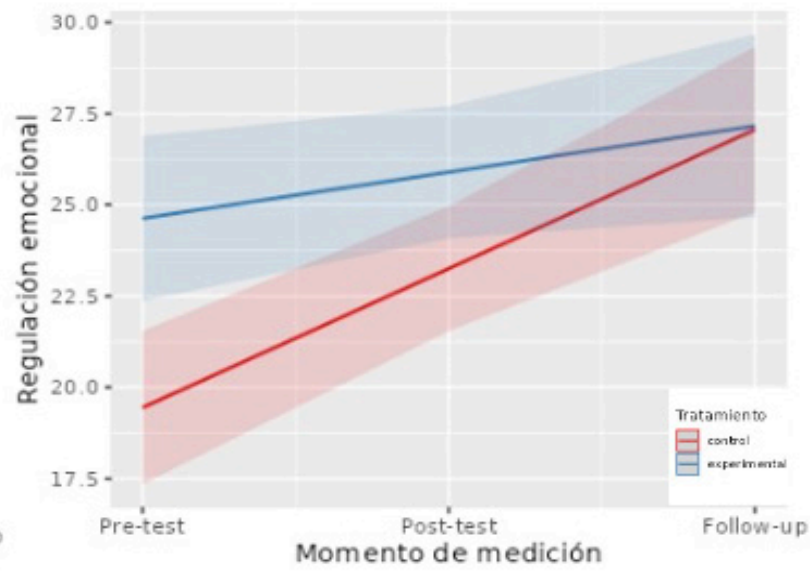


En el grupo experimental, se observa una disminución significativa de los problemas externalizantes a lo largo de los tres momentos de medición. En ambos grupos se observa un aumento en la regulación emocional de mayor magnitud en el grupo control que en el grupo experimental

\section{CONCLUSIONES}

Los resultados preliminares obtenidos mediante modelos de efectos mixtos permitieron identificar que el programa de crianza reflexiva tuvo un efecto significativo, sobre el interés y curiosidad de los cuidadores acerca de los estados mentales de sus hijos, sobre el estrés parental, y sobre los problemas externalizantes de sus hijos tales como gritos, peleas y perder el control. Además, estos efectos se mantuvieron a los tres meses de seguimiento. Este es el primer intento sistemático y científico, para medir este programa en Panamá. Estos hallazgos aportan evidencia sobre la efectividad del Programa Crianza Reflexiva y es la primera vez que se realiza en una población latina. Este tipo de intervención está diseñada para mejorar las habilidades parentales mediante herramientas concretas y accesibles; disiminuye la probabilidad de que un cuidador utilice disciplina violenta y otros tipos de tratos abusivos en niños de nuestro país.

\section{Referencias}

[1] Unicef, "Derecho a una vida libre de violencia," 2019, pp. 77-100.

[2] M. C. Richaud, B. Mesurado, P. Samper-García, A. Llorca, V. Lemos, and A. Tur, "Estilos parentales, inestabilidad emocional y agresividad en niños de nivel socioeconómico bajo en Argentina y España," Ansiedad y Estres, vol. 19, no. 1, 2013.

[3] H. A. Behice, F. Gümüstas, and E. Koyunco Kütük, "Effects of Domestic Violence Against Women on Mental Health of Women and Children," Psikiyatride Guncel Yaklasimlar - Current Approaches in Psychiatry, vol. 12, no. 2, 2020.

[4] C. K. M. Lo and S. Y. Wong, "The effectiveness of parenting programs in regard to improving parental reflective functioning: a meta-analysis," Attachment and Human Development, 2020, doi: 10.1080/14616734.2020.1844247.

[5] T. Fujiwara, N. Kato, and M. R. Sanders, "Effectiveness of Group Positive Parenting Program (Triple P) in Changing Child Behavior, Parenting Style, and Parental Adjustment: An Intervention Study in Japan," Journal of Child and Family Studies, vol. 20, no. 6, pp. 804-813, 2011, doi: 10.1007/s10826011-9448-1.

[6] S. Redfern, "Reflective Parenting Program." Unpublished, London, London, pp. 1-77, 2018.

[7] A. Cooper and S. Redfern, Reflective parenting: A guide to understanding what's going on in your child's mind. 2016.

[8] A. Slade, "Parental reflective functioning: An introduction," Attachment and Human Development. 2005. doi: 10.1080/14616730500245906.

[9] L. S. Sadler et al., "Minding the baby: Enhancing reflectiveness to improve early health and relationship outcomes in an interdisciplinary home-visiting program," Infant Mental Health Journal, 
vol. 34, no. 5, 2013, doi: 10.1002/imhj.21406.

[10] P. Fonagy, M. Target, H. Steele, and M. Steele, "Reflective Functioning Manual version 5.0 for application to AAI," no. July. University College London, London, pp. 1-47, Jul. 1998.

[11] [N. Midgley and I. Vrouva, Minding the child: Mentalization-based interventions with children, young people and their families. 2013. doi: 10.4324/9780203123003.

[12] R. R. Abidin, "Parenting Stress Index 4th Edition," 2012.

[13] M. B. C. Woolgar, S. Falconer, S. Humavun, A. Marsden, S. Scott, and M. Dadds, "A new, brief measure of parental efficacy for parenting practicioners.," Departments of Child and Adolescent Psychiatry: National Academy of Parenting Practicioners: Kings College London., 2012.

[14] The parental reflective functioning questionnaire: Development and preliminary validation," PLoS ONE, vol. 12, no. 5, May 2017, doi: 10.1371/journal.pone.0176218.

[15] A. Goodman and R. Goodman, "Strengths and Difficulties Questionnaire scores and mental health in looked after children," British Journal of Psychiatry, vol. 200, no. 5, 2012, doi: 10.1192/bjp. bp.111.104380.

[16] A. Shields and D. Cicchetti, "Emotion regulation among school-age children: the development and validation of a new criterion Q-sort scale.," Dev Psychol, 1997, doi: 10.1037/0012-1649.33.6.906.

[17] C. M. Carvalho, N. G. Polson, and J. G. Scott, "The horseshoe estimator for sparse signals," Biometrika, vol. 97, no. 2, 2010, doi: 10.1093/biomet/asq017.

[18] F. Wetschoreck, "Rip correlation. Introducing the Predictive Power Score," April 23, 2020. https:// towardsdatascience.com/rip-correlation-introducing-the-predictive-power-score-3d90808b9598 (accessed Jul. 16, 2021).

\section{Autorización y Licencia CC}

Los autores autorizan a APANAC XVIII a publicar el artículo en las actas de la conferencia en Acceso Abierto (Open Access) en diversos formatos digitales (PDF, HTML, EPUB) e integrarlos en diversas plataformas online como repositorios y bases de datos bajo la licencia CC:

Attribution-NonCommercial-ShareAlike 4.0 International (CC BY-NC-SA 4.0) https://creativecommons. org/licenses/by-nc-sa/4.0/.

Ni APANAC XVIII ni los editores son responsables ni del contenido ni de las implicaciones de lo expresado en el artículo. 